\title{
CSF3R T618I, SETBP1 G870S, SRSF2 P95H, and ASXL1 Q780* tetramutation co-contribute to myeloblast transformation in a chronic neutrophilic leukemia
}

\author{
Yi Qian ${ }^{1} \cdot$ Yan Chen ${ }^{1} \cdot$ Xiaoming $\mathrm{Li}^{1}$ \\ Received: 24 October 2020 / Accepted: 8 March 2021 / Published online: 6 April 2021 \\ (C) The Author(s) 2021
}

\begin{abstract}
Chronic neutrophilic leukemia (CNL) is a rare but serious myeloid malignancy. In a review of reported cases for WHO-defined CNL, CSF3R mutation is found in about $90 \%$ cases and confirmed as the molecular basis of CNL. Concurrent mutations are observed in CSF3R-mutated CNL patients, including ASXL1, SETBP1, SRSF2, JAK2, CALR, TET2, NRAS, U2AF1, and CBL. Both ASXL1 and SETBP1 mutations in CNL have been associated with a poor prognosis, whereas, SRSF2 mutation was undetermined. Our patient was a 77-year-old man and had no significant past medical history and symptoms with leukocytosis. Bone marrow (BM) aspirate and biopsy revealed a markedly hypercellular marrow with prominent left-shifted granulopoiesis. Next-generation sequencing (NGS) of DNA from the BM aspirate of a panel of 28 genes, known to be pathogenic in MDS/MPN, detected mutations in CSF3R, SETBP1, and SRSF2, and a diagnosis of CNL was made. The patient did not use a JAK-STAT pathway inhibitor (ruxolitinib) but started on hydroxyurea and alpha-interferon and developed pruritus after 4 months of diagnosis and nasal hemorrhage 1 month later. Then, the patient was diagnosed with CNL with AML transformation and developed intracranial hemorrhage and died. We repeated NGS and found that three additional mutations were detected: ASXL1, PRKDC, MYOM2; variant allele frequency (VAF) of the prior mutations in CSF3R, SETBP1, and SRSF2 increased. The concurrence of CSF3RT618I, ASXL1, SETBP1, and SRSF2 mutation may be a mutationally detrimental combination and contribute to disease progression and AML transformation, as well as the nonspecific treatment of hydroxyurea and alphainterferon, but the significance and role of PRKDC and MYOM2 mutations were not undetermined.
\end{abstract}

Keywords Chronic neutrophilic leukemia $\cdot$ CSF3RT618I $\cdot$ AML transformation

\section{Introduction}

Chronic neutrophilic leukemia $(\mathrm{CNL})$ is a rare but serious myeloid malignancy. Here, we present a case of CNL and discuss the significance of co-expressed mutations in transformation to acute myeloid leukemia (AML). Our patient was a 77-year-old man and had no significant past medical history and symptoms such as fatigue, weight loss, easy bruising, pruritus, abdominal distention, and night sweats. He was hospitalized with chest pain. There was no splenomegaly and

Xiaoming $\mathrm{Li}$

1xm6358@21cn.com

1 Department of Hematology, Affiliated Hospital of Southwest Medical University, Luzhou 646000, Sichuan Province, China hepatomegaly on physical examination. His complete blood count showed white blood cell (WBC) count $88.90 \times 10^{9} / \mathrm{L}$; neutrophil $83.03 \times 10^{9} / \mathrm{L}$; red blood cell $3.45 \times 10^{9} / \mathrm{L}$; hemoglobin $98 \mathrm{~g} / \mathrm{L}$; and platelet $80 \times 10^{9} / \mathrm{L}$. Bone marrow $(\mathrm{BM})$ aspirate and biopsy revealed a markedly hypercellular marrow with prominent left-shifted granulopoiesis without dysplasia and blast proliferation. Reticulin fibrosis was not observed. Cytogenetic analysis was normal. Fluorescence in situ hybridization (FISH) results for the JAK2, FGFR1, FIP1L1PDGFRA, and PDGFRB rearrangement were negative. Polymerase chain reaction results for the BCR-ABL1 fusion, Jak2, MPL, and CALR gene were negative. Next-generation sequencing (NGS) of DNA from the BM aspirate of a panel of 28 genes, known to be pathogenic in MDS/MPN, detected mutations in CSF3R, SETBP1, and SRSF2, and a diagnosis of CNL was made (Tables 1 and 2). 
Table 1 The mutation outcomes at diagnosis of CNL. Positive mutations

\begin{tabular}{|c|c|c|}
\hline Gene & Exon & VAF \\
\hline CSF3R & NM_000760:c.1853>T(p.T618I)exon 14 & $44.1 \%$ \\
\hline SETBPI & NM_015559:c.2608G>(p.G870S)exon4 & $47.1 \%$ \\
\hline $\mathrm{SRSF} 2 \hookleftarrow$ & NM 003016:c.284C>A(p.95H)exon 1↔ & $45.8 \%$ \\
\hline
\end{tabular}

The patient did not use a JAK-STAT pathway inhibitor (ruxolitinib) for CSF3RT618I mutation but started on hydroxyurea and alpha-interferon to control the myeloproliferation. After 7 days of treatment, he had stabilization of the peripheral blood count, with WBC $7.77 \times 10^{9} / \mathrm{L}$, hemoglobin $76 \mathrm{~g} / \mathrm{L}$, and platelet $122 \times 10^{9} /$ L. But he had no regular follow-up and developed pruritus after 4 months of diagnosis and nasal hemorrhage 1 month later. Serum LDH was $1335.2 \mathrm{U} / \mathrm{L}$. His WBC count increased to $128.52 \times 10^{9} / \mathrm{L}$ and hemoglobin and platelets dropped to $68 \mathrm{~g} / \mathrm{dL}$ and $27 \times 10^{9} / \mathrm{L}$, respectively. The peripheral blood smear showed $3 \%$ myeloblasts (Fig. 1a) and BM aspirate revealed $23.5 \%$ myeloblasts and pathological hematopoiesis (Fig. 1b). A diagnosis of CNL with AML transformation was made. We repeated cytogenetic analysis and NGS. Cytogenetic analysis still was normal. FISH results for the BCR-ABL1, ASS1, and +8 were negative. Using the sequencing panel with 96 genes associated with hematopoietic and lymphoid tissue tumors, three additional mutations were detected: ASXL1, PRKDC, MYOM2; variant allele frequency (VAF) of the prior mutations in CSF3R, SETBP1, and SRSF2 increased (Tables 3 and 4). He was admitted for pruritus and nasal hemorrhage, developed intracranial hemorrhage, and expired.

In a review of reported cases for WHO-defined CNL, CSF3R mutation is found in about $90 \%$ cases [1] and confirmed as the molecular basis of CNL. Concurrent mutations are observed in patients with CSF3Rmutated CNL, including ASXL1, SETBP1, SRSF2, JAK2, CALR, TET2, NRAS, U2AF1, and CBL [2-7]. Elliott et al. found that presence of ASXL1 mutation

Table 2 The mutation outcomes at diagnosis of CNL. Negative mutations

\begin{tabular}{llllll}
\hline ASXL1 & BCOR & CALR & CBL $\hookleftarrow$ & DNMT3A & ETV6 \\
JAK3 $\hookleftarrow$ & MPL $\hookleftarrow$ & NPM1 & NRAS & PHF6 & PTPN11 \\
RUNX1 $\hookleftarrow$ & SF3B1 & STAG2 & TET2 & TP53 & U2AF1 \\
ZRSR2 & $\hookleftarrow$ & $\hookleftarrow$ & $\hookleftarrow$ & $\hookleftarrow$ & $\hookleftarrow$ \\
\hline
\end{tabular}

Table 3 The mutation outcomes at AML transformation. Positive mutations

\begin{tabular}{|c|c|c|}
\hline Gene & Exon & $\mathrm{VAF} \hookleftarrow$ \\
\hline ASXL1 & NM_015338:c.2338C>T(p.Q780*)exon12 & $46.1 \%$ \\
\hline CSF3R & NM_000760:c.1853C>T(p.T618I)exon14 & $49.6 \%$ \\
\hline SETBP1 & NM_015559:c.2608G>A(p.G870S)exon4 & $48.6 \%$ \\
\hline SRSF2 & NM_003016:c.284C>A(p.P95H)exon1 & $49.7 \%$ \\
\hline PRKDC $\hookleftarrow$ & NM_006904:c.9921+1G>T exon70 & $4 \%$ \\
\hline MYOM2 & NM_003970:c.3901T>C(p.Y1301H)exon33 & $51 \%$ \\
\hline
\end{tabular}

and thrombocytopenia in CSF3R-mutated CNL were independently predictive of shortened survival on multivariable analysis [2]. Two patients with SETBP1mutated and ASXL1-unmutated developed AML transformation, whereas two other patients with ASXL1mutated and SETBP1-unmutated evolved into chronic myelomonocytic leukemia (CMML) in their study. Both ASXL1 mutation and SETBP1 mutation in CNL have been associated with a poor prognosis. SRSF2 mutation has been frequently reported in myelodysplastic syndromes (MDS) and CMML patients which is related to shorter OS and may be considered as an adverse prognostic risk factor in MDS, but not in CMML [8], however, which was undetermined in CNL. In our case, ASXL1 mutation (along with thrombocytopenia) was detected with PRKDC and MYOM2 mutations without the sequencing panel at preliminary diagnosis at low levels, and VAF of CSF3RT618I, SETBP1, and SRSF2 increased at progression to AML transfor-

Table 4 The mutation outcomes at AML transformation. Negative mutations

\begin{tabular}{llllll}
\hline ABCB1 & ABL1 & ANKRD26 & APC & ARID1A & ARID1B \\
ARID2 & ATG2B & ATM & ATRX & B2M & BCL10 \\
BCL2 & BCL6 & BCOR & BCORL1 & BIRC3 & BLM \\
BPGM & BRAF & BRCA1 & BRCA2 & BRIP1 & BTG1 \\
BTK & CALR & CARD11 & CBL & CBLC & CBLC \\
CCND1 & CCND3 & CD28 & CD58 & CD79A & CD79B \\
CDKN1A & CDKN2A & CDKN2B & CEBPA & CHD8 & CIITA \\
CREBBP & CRLF2 & CSF1R & CTCF & CUX1 & CXCR4 \\
DDX41 & DIS3 & DKC1 & DNM2 & DNMT3A & EED \\
EGFR & EGLN1 & ELANE & EP300 & EPHA7 & EPOR \\
ETV6 & EZH2 & FAM46C & FAS & FAT1 & FBXO11 \\
FBXW7 & FLT3 & FOXO1 & GATA1 & GATA2 & GFI1 \\
GNA13 & GNAI2 & GNAS & GNB1 & GSKIP & HAXI \\
HRAS & ID3 & IDH1 & IDH2 & IKZF1 & IKZF2 \\
IKZF3 & IL7R & IRF4 & IRF8 & ITPKB & JAK1 \\
\hline
\end{tabular}


Fig. 1 a Peripheral blood (Giemsa, $\times 200)$. b Bone marrow aspirate (Giemsa, $\times 400)$

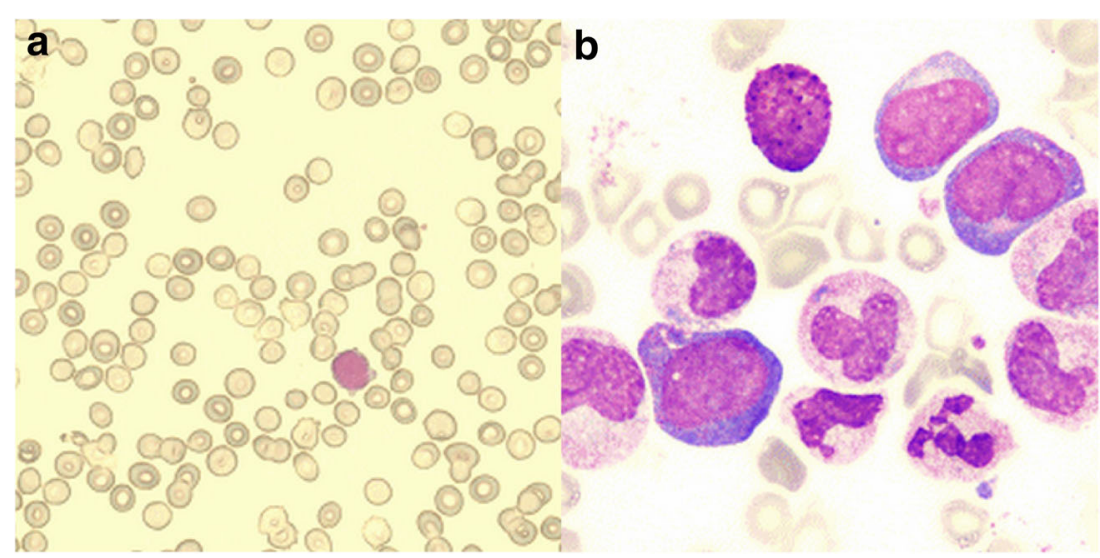

mation. It suggested that concurrence of CSF3RT618I, ASXL1, SETBP1, and SRSF2 mutation may be a mutationally detrimental combination and contribute to disease progression and AML transformation, as well as the nonspecific treatment of hydroxyurea and alpha-interferon, but the significance and role of PRKDC and MYOM2 mutations were not undetermined.

In conclusion, we report here that concurrence of CSF3RT618I, ASXL1, SETBP1, and SRSF2 mutations may co-contribute to AML transformation, but their interactions with PRKDC and MYOM2 mutations in the pathogenesis of CNL as well as their prognostic implications remain to be determined.

\section{Declarations}

Ethical approval All procedures performed in studies involving human participants were in accordance with the ethical standards of the institutional and/or national research committee and with the 1964 Helsinki Declaration and its later amendments or comparable ethical standards.

Informed consent Written informed consent was obtained from the patient for the publication of this case report and any accompanying images.

Conflict of interest The authors declare no competing interests.

Open Access This article is licensed under a Creative Commons Attribution 4.0 International License, which permits use, sharing, adaptation, distribution and reproduction in any medium or format, as long as you give appropriate credit to the original author(s) and the source, provide a link to the Creative Commons licence, and indicate if changes were made. The images or other third party material in this article are included in the article's Creative Commons licence, unless indicated otherwise in a credit line to the material. If material is not included in the article's Creative Commons licence and your intended use is not permitted by statutory regulation or exceeds the permitted use, you will need to obtain permission directly from the copyright holder. To view a copy of this licence, visit http://creativecommons.org/licenses/by/4.0/.

\section{References}

1. Pardanani A, Lasho TL, Laborde RR, Elliott M, Hanson CA, Knudson RA, Ketterling RP, Maxson JE, Tyner JW, Tefferi A (2013) CSF3R T618I is a highly prevalent and specific mutation in chronic neutrophilic leukemia. Leukemia. 27(9):1870-1873

2. Elliott MA, Pardanani A, Hanson CA, Lasho TL, Finke CM, Belachew AA, Tefferi A (2015) ASXL1 mutations are frequent and prognostically detrimental in CSF3R-mutated chronic neutrophilic leukemia. Am J Hematol 90(7):653-656

3. Cui Y, Li B, Gale RP, Jiang Q, Xu Z, Qin T, Zhang P, Zhang Y, Xiao $Z$ (2014) CSF3R, SETBP1 and CALR mutations in chronic neutrophilic leukemia. J Hematol Oncol 7:77

4. Price A, Druhan LJ, Lance A, Clark G, Vestal CG, Zhang Q, Foureau D, Parsons J, Hamilton A, Steuerwald NM, Avalos BR (2020) T618I CSF3R mutations in chronic neutrophilic leukemia induce oncogenic signals through aberrant trafficking and constitutive phosphorylation of the O-glycosylated receptor form. Biochem Biophys Res Commun 523(1):208-213

5. Maxson JE, Tyner JW (2017) Genomics of chronic neutrophilic leukemia. Blood. 129(6):715-722

6. Zhang Y, Wang F, Chen X, Zhang Y, Wang M, Liu H, Cao P, Ma X, Wang T, Zhang J, Zhang X, Lu P, Liu H (2018) CSF3R mutations are frequently associated with abnormalities of RUNX1, CBFB, CEBPA, and NPM1 genes in acute myeloid leukemia. Cancer. 124(16):3329-3338

7. Guo YJ, Wang Y, Wang LH, Zuo YB, Niu ZY, Lin FR, Zhang JY (2020) Analysis of clinical features and prognosis of patients with chronic neutrophil leukemia. Zhongguo Shi Yan Xue Ye Xue Za Zhi 28(1):82-87

8. Arbab JP, Ayatollahi H, Sadeghi R, Sheikhi M, Asghari A (2018) Prognostic significance of SRSF2 mutations in myelodysplastic syndromes and chronic myelomonocytic leukemia: a meta-analysis. Hematology. 23(10):778-784

Publisher's note Springer Nature remains neutral with regard to jurisdictional claims in published maps and institutional affiliations. 